\title{
Sizes of Anode and Cathode Affect Electricity Generation in Rice Paddy-Field Microbial Fuel Cells
}

\author{
Nagayoshi Ueoka1 ${ }^{*}$, Naoko Sese ${ }^{1 *}$, Mayu Sue ${ }^{2}$, Atsushi Kouzuma1, Kazuya Watanabe ${ }^{1 \#}$ \\ ${ }^{1}$ School of Life Sciences, Tokyo University of Pharmacy and Life Sciences, Tokyo, Japan \\ ${ }^{2}$ Technical Research Institute, Maeda Corporation, Tokyo, Japan \\ Email: "kazuyaw@toyaku.ac.jp
}

Received 6 February 2016; accepted 14 March 2016; published 17 March 2016

Copyright (C) 2016 by authors and Scientific Research Publishing Inc.

This work is licensed under the Creative Commons Attribution International License (CC BY). http://creativecommons.org/licenses/by/4.0/

(c) (i) Open Access

\begin{abstract}
Rice paddy-field microbial fuel cells (RPF-MFCs) are devices that exploit rhizosphere bacteria to generate electricity from soil organic matter, including those excreted from roots. Previous studies have examined factors affecting electric outputs from RPF-MFCs and demonstrated that RPFMFC was able to generate electricity up to $80 \mathrm{~mW} \cdot \mathrm{m}^{-2}$ (based on the projected area of anode). The present study operated RPF-MFCs with different sizes of anodes and cathodes and examined how electrode sizes affected electricity generation. We show that anodes are the limiting factor for electricity generation immediately after commencing the operation, while cathodes become the limiting factor after anode performances are sufficiently increased. RPF-MFC achieved the maximum power density of $140 \mathrm{~mW} \cdot \mathrm{m}^{-2}$ (based on the projected area of anode), when the cathode is sufficiently larger than the anode. Results suggest that the cathode needs to be improved for eliciting the maximum capacity of rhizosphere bacteria for electricity generation in RPF-MFC.
\end{abstract}

\section{Keywords}

Root Exudates, Polarization Analyses, Pyrosequencing, 16S rRNA Gene, Geobacter

\section{Introduction}

Rice paddy-field microbial fuel cells (RPF-MFCs, Figure 1) are sediment-type MFCs that convert soil organic matter into electricity with the aid of rhizosphere microbes [1] [2]. Different from other sediment-type MFCs [3],

\footnotetext{
"These authors equally contributed to the study.

${ }^{*}$ Corresponding author.
} 


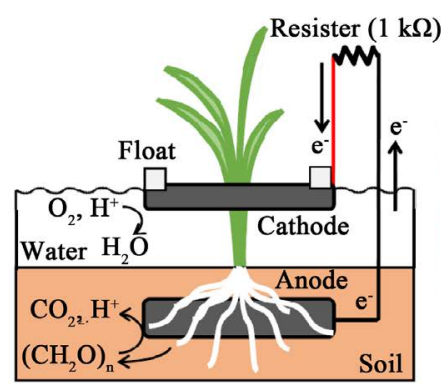

(a)

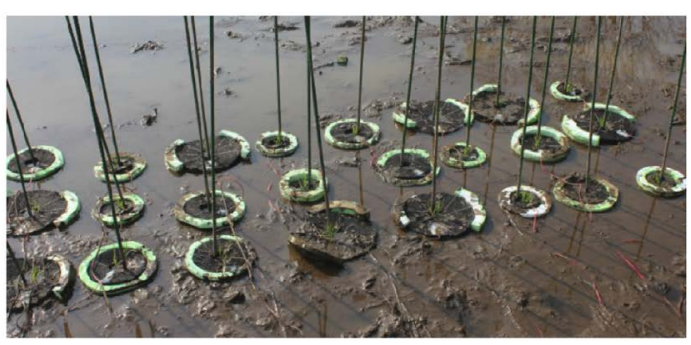

(b)

Figure 1. RPF-MFC used in the present study. (a) Systematic diagram showing the configuration of RPF-MFC; (b) Photo of RPF-MFCs immediately after commencing the experiment.

RPF-MFCs utilize organic matter excreted from rice roots (root exudates [4]) for electricity generation. RPFMFCs are expected to be applied to on-site power sources for remote sensors that facilitate smart agriculture. A previous study has shown that rice roots excrete sugars (e.g., glucose) and organic acids (e.g., acetate) that can be converted to electricity by anode-associated microbes [5]. Another study combined phylogenetic and metagenomic approaches for characterizing anode-associated microbes, suggesting that bacteria affiliated with the genus Geobacter (particularly those closely related to G. pelophilus and G. psychrophilus) were involved in electricity generation in RPF-MFCs [6]. Another previous study has examined factors affecting electricity generation in RPF-MFCs and found that cathode modification with platinum catalysts, anode position and external load largely affected power outputs [7]. In addition, it has also been found that graphite-felt cathodes equipped with polystyrene-foam floaters enable relatively stable and high power outputs from RPF-MFCs [2]. Based on these examinations, the maximum power and short-circuit current densities have reached $80 \mathrm{~mW} \cdot \mathrm{m}^{-2}$ and 550 $\mathrm{mA} \cdot \mathrm{m}^{-2}$, respectively (based on the projected anode area) [2]. Compared to the maximum power density (6 $\left.\mathrm{mW} \cdot \mathrm{m}^{-2}\right)$ reported in the first trial of RPF-MFCs in 2008 [5], the latest power density $\left(80 \mathrm{~mW} \cdot \mathrm{m}^{-2}\right)$ has increased over 10-fold, showing that the RPF-MFC technology has progressed substantially.

Despite the substantial improvement achieved in the recent studies [2] [7], there still exist unexamined factors that may be important for further increasing electric outputs from RPF-MFCs. In the present work, we attempted to identify which electrode (namely, anode or cathode) is the limiting factor for electricity generation in RPF-MFC. For this purpose, RPF-MFCs were equipped with different sizes of anodes and cathodes, and power outputs were measured during the operation.

\section{Materials and Methods}

\subsection{RPF-MFC Setups}

An area of the Egawa rice paddy field (Noda Natural Symbiotic Farm Co.) was used for RPF-MFC experiments. The configuration of the RPF-MFC system was similar to that described previously [6], and a single electrode system was set for each rice-plant hill (Figure 1). The anode and cathode were made of graphite felt (GF-80-5F, Sohgoh Carbon) and had different projected areas as summarized in Table 1. Each RPF-MFC type had five replicates. Electrodes are circular, and each had a single hole $(10 \mathrm{~cm}$ in diameter) at the center for planting of rice plants. A platinum catalyst (TEC10E20A, Tanaka Kikinzoku Hanbai) was loaded on the graphite felt cathode at a density of $0.1 \mathrm{mg} \cdot \mathrm{cm}^{-2}$ using nafion as a binder. The anode and cathode were connected via epoxy encapsulated wires, and the circuit was completed using an external resister (1000 $\Omega$ ). Rice-plant seedlings (Oryza sati$v a$ L. cv. Koshihikari) were transplanted on April 25, 2015 (day 0) and cultivated using standard procedures [5].

\subsection{Polarization Analyses}

Polarization curves (current vs. voltage) were plotted using a potentiostat (HSV-100, Hokuto Denko) and used to draw power curves (current vs. power). As an index for the electrochemical capacity of RPF-MFC, we determined the maximum power (the peak of a power curve; $P_{\max }[\mathrm{mW}]$ ) [8]. Using $P_{\max }$ values, the maximum power densities per projected areas of the anode or cathode $\left(Q_{\max }\left[\mathrm{mW} \cdot \mathrm{m}^{-2}\right]\right)$ were calculated. Data were statistically analyzed by the $t$ test $(\mathrm{P}<0.05)$. 
Table 1. Electrode sizes of RPF-MFC systems used in the present study.

\begin{tabular}{ccc}
\hline \multirow{2}{*}{ RPF-MFC System } & Size $\left(\mathrm{cm}^{-2}\right)$ & \\
\cline { 2 - 3 } SE & Anode & Cathode \\
LE & 50 & 50 \\
AL & 424 & 424 \\
CL & 50 & 424 \\
\hline
\end{tabular}

\subsection{Analyses of Anode Microbiomes}

DNA was extracted from bulk paddy-field soil $(0.5 \mathrm{~g})$ and anode graphite-felt pieces $(0.5 \times 0.5 \mathrm{~cm})$ that were sampled on day 93. For anode samples, surface felts and loosely attached soil particles were removed, and core felt samples were used for DNA extraction. DNA was extracted using a Fast DNA SPIN Kit for Soil (Funakoshi) according to manufacturer's instructions, and purified DNA was finally dissolved in $50 \mu \mathrm{L}$ of the DES solution supplied in the kit. PCR amplification of 16S rRNA gene fragments (V1-V3 region) was performed using primers ad-tag-8F and ad-533R [6]. The PCR mixture and thermal cycling conditions were as described elsewhere [9]. Amplicons were purified using a QIA quick PCR purification kit (Qiagen) and mixed at the same concentration $\left(1 \mathrm{ng} \cdot \mu \mathrm{L}^{-1}\right.$ each). The mixed amplicons were subjected to pyrosequencing using a Genome Sequencer FLX system (Roche Applied Science). Phylogenetic analyses were conducted using the Silva rRNA database http://www.arb-silva.de/, and a tree was constructed by the neighbor-joining method using MEGA5 [10]. To evaluate the robustness of the inferred tree, the bootstrap resampling method [11] was used with 100 replicates. Nucleotide sequences determined in the present study has been deposited into the DDBJ Sequence Read Archive Database (accession number: DRA004371).

\section{Results and Discussion}

In order to examine effects of anode and cathode performances on power outputs from the total RPF-MFC system, RPF-MFCs with different sizes of anodes and cathodes (Table 1) were operated, and their power indices ( $P_{\max }$ and $Q_{\max }$ values) were compared (Figure 2). For a reactor-type MFC, polarization analyses using a reference electrode is possible for evaluating electrochemical performances of anodes and cathodes [8]. However, since a reference electrode did not work in soil, we employed the method in which RPF-MFC systems with different sizes of anodes and cathodes were comparatively analyzed. The operation of the RPF-MFCs was initiated when rice-plant seedlings were transplanted and terminated when rice crops were harvested (30 August). During the operation, polarization measurements were conducted several times to examine temporal changes in power outputs (Figure 2).

We found that LE generated larger power than SE (Figure 2(a)). Power densities for LE were however smaller than those for SE (Figure 2(b) and Figure 2(c)), probably because more organics were supplied from rice roots to anode areas close to rice plants than those far from plants. We also found that $P_{\max }$ values for SE and LE were not dependent on operational periods (Figure 2(a)). On the other hand, $P_{\max }$ values for the AL and CL systems were dependent on operational periods; namely, $P_{\max }$ for AL on day 93 was much higher than that on day 20, while vice versa for CL. These data indicate that the anode performance increases during the operation of RPF-MFC, while the cathode performance decreases. It is likely that the anode improvement and cathode deterioration similarly affected the $P_{\max }$ values in the SE and LE systems, resulting in the relatively stable $P_{\max }$ values.

In the present study, the highest $Q_{\max }$ value (approximately $140 \mathrm{~mW} \cdot \mathrm{m}^{-2}$ ) was observed for the $\mathrm{AL}$ system on day 93 (Figure 2(b)). This value for the AL system is much higher than that for the SE system, even though these MFC had the same size of anode. In addition, this is the highest $Q_{\max }$ among values (based on the projected area of anodes) so far reported for RPF-MFCs (refer to Ref. 2 for the summary of previous data), suggesting that the large cathode is effective for enhancing the anode-based $Q_{\max }$ value. It is therefore concluded that the cathode needs to be improved for eliciting the maximum capacity of rhizosphere bacteria for generating electricity at the anode of RPF-MFC. 


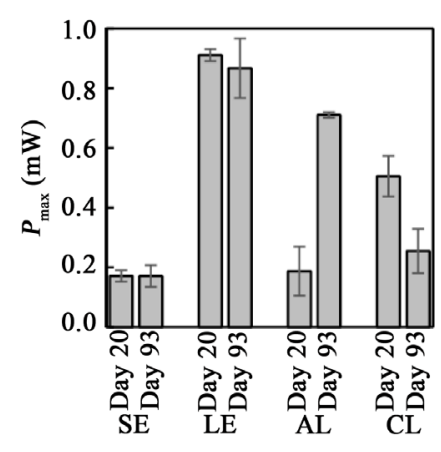

(a)

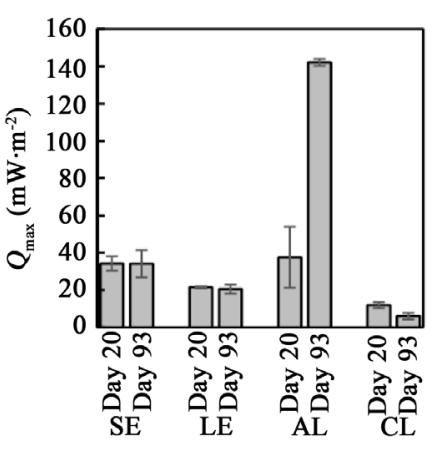

(b)

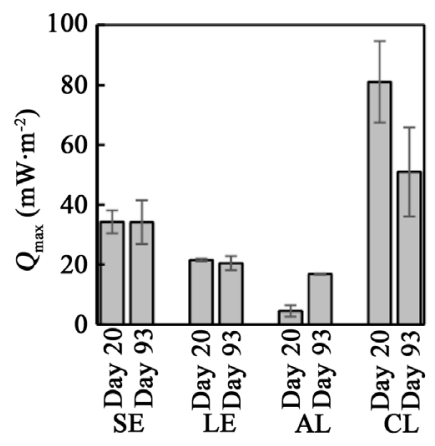

(c)

Figure 2. Evaluation of power outputs from the four systems of RPF-MFC. (a) $P_{\max }$ for the total systems; (b) $Q_{\max }$ based on the anode projected area; (c) $Q_{\max }$ based on the cathode projected area. An error bar indicates SD.

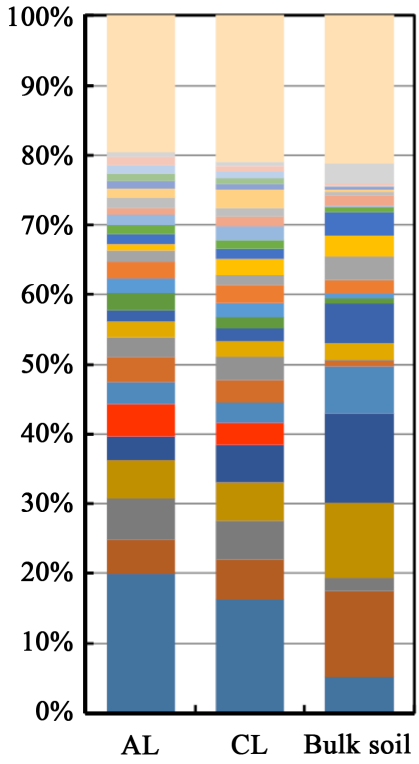

(a)

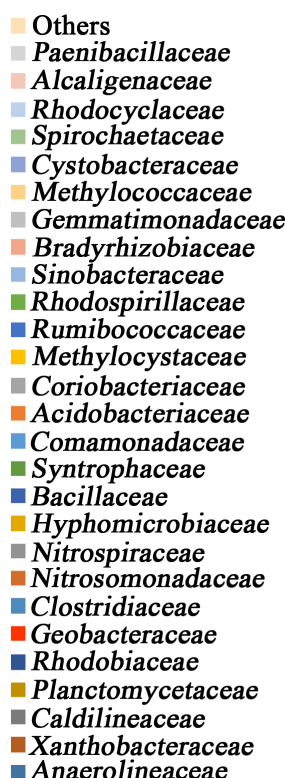

Anthobacteracea

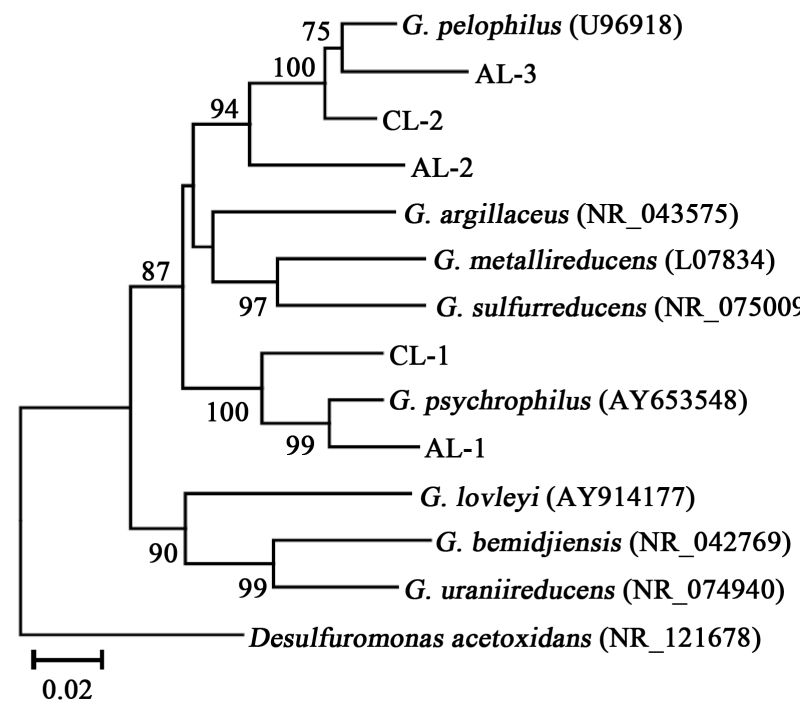

(b)

Figure 3. Phylogenetic analyses of microbiomes associated with anodes of the AL and CL systems and that in bulk soil. (a) Ratios in major families of bacteria; (b) Phylogenetic tree showing relationships among major sequences $(\mathrm{n}>5)$ detected in the AL- and CL-anode microbiomes and representative Geobacter species. Accession numbers are indicated in parentheses. Numbers at branch nodes are bootstrap values (100 trials, only $>50$ values are shown).

Previous studies have suggested that bacteria affiliated with the genus Geobacter are involved in electron transfer to anodes in RPF-MFC systems [6] and plant-associated MFCs in pot-culture systems [12] [13]. Comparative metagenomics of anode-associated microbiomes showed that bacteria closely related to G. pelophilus and G. psychrophilus are involved in electricity generation [6]. In order to examine if Geobacter bacteria also occurred in anode-associated microbiomes, the present study conducted phylogenetic analyses of 16S rRNA gene fragments PCR-amplified from anode microbiomes. Figure 3(a) presents phylogenetic distributions at the family level of bacteria in bulk soil and those in anode-associated microbiomes in the AL and CL systems. It is shown that, compared to Geobacteraceae bacteria in the bulk soil $(<0.1 \%)$, those in the AL $(2.4 \%)$ and CL $(1.8 \%)$ anode samples increased substantially. Comparative analyses of Geobacteraceae sequences retrieved in the present study and those in the databases indicate that those closely related to G. pelophilus [14] and G. psychrophilus [15] were major members in the AL and CL anode sample (Figure 3(b)), suggesting that these Geobacter bacteria were involved in electricity generation in the RPF-MFC system. This result therefore supports the previous finding that G. pelophilus and G. Psychrophilus relatives play important roles in electricity generation in RPF-MFC. 


\section{Conclusion}

The present study suggests that the cathode is the limiting factor for electricity generation in RPF-MFC after anode microbiomes are sufficiently enriched. The cathode performance is found to be decreased during the operation, probably owing to deterioration of the cathode catalysts. In order to sustainably improve the cathode performance, future studies will develop methods to effectively utilize biocathodes [16] [17] for RPF-MFC.

\section{Acknowledgements}

We thank Noda Natural Symbiotic Farm Co. for managing the rice paddy field. We also thank Ayako Matsuzawa and Nanako Amano for technical assistance.

\section{References}

[1] Watanabe, K. and Nishio, K. (2010) Electric Power from Rice Paddy Fields. In: Nathwani, J. and Ng, A., Eds., Paths to Sustainable Energy, InTech, Rijeka, 563-580. http://dx.doi.org/10.5772/12929

[2] Kouzuma, A., Kaku, N. and Watanabe, K. (2014) Microbial Electricity Generation in Rice Paddy Fields: Recent Advances and Perspectives in Rhizosphere Microbial Fuel Cells. Applied Microbiology and Biotechnology, 98, 95219526. http://dx.doi.org/10.1007/s00253-014-6138-0

[3] Reimers, C.E., Tender, L.M., Fertig, S. and Wang, W. (2001) Harvesting Energy from the Marine Sediment-Water Interface. Environmental Science and Technology, 35, 192-195. http://dx.doi.org/10.1021/es001223s

[4] Bais, H.P., Weir, T.L., Perry, L.G., Gilroy, S. and Vivanco, J.M. (2006) The Role of Root Exudates in Rhizosphere Interactions with Plants and Other Organisms. Annual Reviews for Plant Biology, 57, 233-266. http://dx.doi.org/10.1146/annurev.arplant.57.032905.105159

[5] Kaku, N., Yonezawa, N., Kodama, Y. and Watanabe, K. (2008) Plant/Microbe Cooperation for Electricity Generation in a Rice Paddy Field. Applied Microbiology and Biotechnology, 79, 43-49. http://dx.doi.org/10.1007/s00253-008-1410-9

[6] Kouzuma, A., Kasai, T., Nakagawa, G., Yamamuro, A., Abe, T. and Watanabe, K. (2013) Comparative Metagenomics of Anode-Associated Microbiomes Developed in Rice Paddy-Field Microbial Fuel Cells. PLoS ONE, 8, e 77443. http://dx.doi.org/10.1371/journal.pone.0077443

[7] Takanezawa, K., Nishio, K., Kato, S., Hashimoto, K. and Watanabe, K. (2010) Factors Affecting Electric Output from Rice-Paddy Microbial Fuel Cells. Bioscience, Biotechnology, and Biochemistry, 74, 1271-1273. http://dx.doi.org/10.1271/bbb.90852

[8] Logan, B.E., Hamelers, B., Rozendal, R., Schröder, U., Keller, J., Freguia, S., Aelterman, P., Verstraete, W. and Rabaey, K. (2006) Microbial Fuel Cells: Methodology and Technology. Environmental Science and Technology, 40, 5181-5192. http://dx.doi.org/10.1021/es0605016

[9] Miyahara, M., Hashimoto, K. and Watanabe, K. (2013) Use of Cassetteelectrode Microbial Fuel Cell for Wastewater Treatment. Journal of Bioscience and Bioengineering, 115, 176-181. http://dx.doi.org/10.1016/j.jbiosc.2012.09.003

[10] Tamura, K., Peterson, D., Peterson, N., Stecher, G., Nei, M. and Kumar, S. (2011) MEGA5: Molecular Evolutionary Genetics Analysis Using Maximum Likelihood, Evolutionary Distance, and Maximum Parsimony Methods. Molecular Biology and Evolution, 28, 2731-2739. http://dx.doi.org/10.1093/molbev/msr121

[11] Felsenstein, J. (1985) Confidence Limits on Phylogenies: An Approach Using the Bootstrap. Evolution, 39, $783-791$. http://dx.doi.org/10.2307/2408678.

[12] De Schamphelaire, L., Cabezas, A., Marzorati, M., Friedrich, M.W., Boon, N. and Verstraete, W. (2010) Microbial Community Analysis of Anodes from Sediment Microbial Fuel Cells Powered by Rhizodeposits of Living Rice Plants. Applied and Environmental Microbiology, 76, 2002-2008. http://dx.doi.org/10.1128/AEM.02432-09

[13] Timmers, R.A., Rothballer, M., Strik, D.P., Engel, M., Schulz, S., Schloter, M., Hartmann, A., Hamelers, B. and Buisman, C. (2012) Microbial Community Structure Elucidates Performance of Glyceria Maxima Plant Microbial Fuel Cell. Applied Microbiology and Biotechnology, 94, 537-548. http://dx.doi.org/10.1007/s00253-012-3894-6

[14] Straub, K.L. and Buchholz-Cleven, B.E. (2001). Geobacter bremensis Sp. Nov. and Geobacter pelophilus Sp. Nov., Two Dissimilatory Ferric-Iron-Reducing Bacteria. International Journal of Systematic and Evolutionary Microbiology, 51, 1805-1808. http://dx.doi.org/10.1099/00207713-51-5-1805.

[15] Nevin, K.P., Holmes, D.E., Woodard, T.L., Hinlein, E.S., Ostendorf, D.W. and Lovley, D.R. (2005) Geobacter bemidjiensis Sp. Nov. and Geobacter psychrophilus Sp. Nov., Two Novel Fe(III)-Reducing Subsurface Isolates. International Journal of Systematic and Evolutionary Microbiology, 55, 1667-1674. http://dx.doi.org/10.1099/ijs.0.63417-0 
[16] He, Z. and Angenent, L.T. (2006) Application of Bacterial Biocathodes in Microbial Fuel Cells. Electroanalysis, 18, 2009-2015. http://dx.doi.org/10.1002/elan.200603628

[17] De Schamphelaire, L., Rabaey, K., Boeckx, P., Boon, N. and Verstraete, W. (2008) Outlook for Benefits of Sediment Microbial Fuel Cells with Two Bio- Electrodes. Microbial Biotechnology, 1, 446-462.

http://dx.doi.org/10.1111/j.1751-7915.2008.00042.x 